\title{
Response Function of the Fractional Quantized Hall State on a Sphere II: Exact Diagonalization
}

\author{
Song He \\ AT\&T Bell Laboratories, Murray Hill, NJ 07974 \\ S. H. Simon and B. I. Halperin \\ Department of Physics, Harvard University \\ Cambridge, MA 02138
}

(Feb 21, 1994)

\begin{abstract}
We study the excitation spectra and the dynamical structure factor of quantum Hall states in a finite size system through exact diagonalization. Comparison is made between the numerical results so obtained and the analytic results obtained from a modified RPA in the preceding companion paper [何]. We find good agreement between the results at low energies.
\end{abstract}

Typeset using REVTEX 


\section{INTRODUCTION}

By using a singular gauge transformation to convert electrons into fermions interacting through a Chern-Simons field, much progress has been made in understanding the physics of a two-dimensional interacting electron systems in strong magnetic fields at simple filling fractions [1] 3]. At the mean field level, this transformation maps certain fractional quantized Hall states into integer quantized Hall states of transformed fermions so that some conventional many-body techniques may be used to tackle the problem. In the preceding companion paper [1], an analytic procedure call the modified random phase approximation (modified RPA) has been constructed in the spherical geometry for evaluating the response of certain quantized Hall states to external perturbations. The purpose of this paper is to present numerical results of excitation spectra and the dynamical structure factors at selected filling fractions, calculated using exact diagonalization and the modified RPA, so that comparison can be made and the performance of the modified RPA can be evaluated. Specifically, we present numerical results from both exact diagonalization and modified RPA for systems of $N=8$ and $N=12$ electrons at filling factor $\nu=1 / 3$ and $\nu=3 / 7$ respectively.

As usual for numerical calculations, we work in the limit where the electron-electron interaction is weak compared to the cyclotron energy. For the exact diagonalization method, this means that all electrons are confined to the lowest Landau level. Within the modified RPA calculations, this means that we are working in the limit where the bare band mass $m_{b}$ is infinitely small compared to the effective mass $m^{*}$, whose scale is set by the electronelectron interaction strength. (In practice, we have carried out these calculations for the value $\left.m^{*} / m_{b}=50.\right)$

Our main purpose is to compare the exact and approximate calculations of the dynamical structure factor $S(q, \omega)$ over a range of wavevectors, for the frequencies which are on the scale of the electron-electron energy, and therefore very much lower than the cyclotron frequency $\omega_{c}$. For such frequencies, the structure factor $S(q, \omega)$ is equivalent to the projected structure factor $\bar{S}(q, \omega)$, in the exact calculation where all electron operators are projected 
onto the lowest Landau level. To obtain additional information, however, we wish to compare various frequency moments of the projected structure factor with the corresponding quantities obtained from the modified RPA. (Specifically, we calculate contributions to the $f$-sum rule, the projected static structure factor $\bar{S}(q)$, and the static wavevector-dependent compressibility.) For these comparisons, we define the projected structure factor in the modified RPA by excluding the contribution of the "Kohn mode", which occurs at the cyclotron frequency at $q \approx 0$.

Our plan for this paper is as follows. In section II, we describe the model and briefly discuss the method we use in the exact diagonalization. In addition, we review a few necessary points of the modified RPA calculation. In section III, we present results for the projected dynamical structure factor $\bar{S}(q, \omega)$ which describes the low energy response of the system to an external perturbation and gives the dispersion of the collective modes of the system. As well as showing $\bar{S}(q, \omega)$, we will also calculate the dispersion of the collective mode in the single mode approximation (SMA), the contributions of the excitations in the lowest Landau level to the projected static structure factor $\bar{S}(q)$, the $f$-sum rule, and the compressibility sum rule. Our conclusions are summarized in section $\mathbb{\square}$.

\section{MODEL AND METHOD}

We will use the spherical geometry throughout this paper, so our system is given by a magnetic monopole of total flux $N_{\phi}$ quanta at the center of the sphere with $N$ electrons confined to the sphere's surface, interacting via a Coulomb potential. If the system were infinitely large (ie, a planar system), the filling fraction would be given by the ratio of the

number of electrons to the number of flux quanta $\left(\nu=\frac{N}{N_{\phi}}\right)$. However, in a finite sized system, this relation is changed to [4 6

$$
N_{\phi}=[\nu]^{-1} N-X(\nu)
$$

where $X(\nu)$ is known as the "shift" [7] of the state, and depends also on the topology of the system. In this work, we will be interested in filling fractions of the form $\nu_{p}=\frac{p}{2 p+1}$. For 
these states, in our spherical geometry, the shift is given by

$$
X\left(\nu_{p}\right)=2+p
$$

In the spherical geometry, by rotational invariance, the eigenstates of the system can be classified by the conserved quantum numbers $(L, M)$, where $L$ is the quantum number for the total angular momentum of an eigenstate and $M$ is its $z$-component. In order to compare results on a finite sphere with those for an infinite planar system, we identify the wavevector $q$ on the plane with the quantity $L / R$, where $R$ is the radius of the sphere. This identification is exact in the limit $R \rightarrow \infty$, but it is to some degree arbitrary for any finite system. (For example, the identification $q \rightarrow \sqrt{L(L+1)} / R$ would be equally valid.)

In keeping with the convention of the preceding companion paper [1], we choose units of length such that the radius of the sphere is unity. The effective "size" of the sphere is then defined by the magnetic length $l_{0}=S^{-1 / 2}$ where $S=N_{\phi} / 2$ is half the total number

of flux quanta through the surface of the sphere. It is also convenient to choose the unit of energy to be $e^{2} / \epsilon l_{0}$ which is the only energy scale in the problem when all of the electrons are confined to the lowest Landau level.

\section{A. Exact Diagonalization}

Since exact diagonalization in the spherical geometry is a well established method, we will only describe it very briefly; more information can be found in Refs. [4] and [5]. The extraction of the projected dynamical structure factor $\bar{S}(q, \omega)$ from such diagonalizations, however, is a method new to this work.

As mentioned in the preceding companion paper [1], the single particle energy eigenstates are the monopole spherical harmonics $[8] Y_{l, m}^{S}(\Omega)$, where $l=S, S+1, \ldots$, and $m=-l, \ldots, l$. The corresponding eigenenergies are given by

$$
E_{l}=\frac{1}{2 S}\left[l(l+1)-S^{2}\right] \hbar \omega_{c}
$$


where $\omega_{c}=\hbar S / m_{b}$ is the cyclotron frequency, with $m_{b}$ the band mass of the electron. The lowest Landau level has a basis of the $2 S+1$ states with $l=S$. With the single particle states given, ignoring the constant kinetic energy, the Hamiltonian of the many electron system, projected to the lowest Landau level, is written in second quantized notation as

$$
\hat{H}=\frac{1}{2} \sum V_{m_{1}^{\prime}, m_{2}^{\prime} ; m_{1}, m_{2}} C_{S, m_{1}^{\prime}}^{\dagger} C_{S, m_{2}^{\prime}}^{\dagger} C_{S, m_{2}} C_{S, m_{1}},
$$

where $C_{l, m}^{\dagger}$ or $C_{l, m}$ creates or annihilates an electron in the single particle eigenstate $Y_{l, m}^{S}(\Omega)$, and $V_{m_{1}^{\prime}, m_{2}^{\prime} ; m_{1}, m_{2}}$ is the matrix element of the spherical Coulomb interaction $V\left(\boldsymbol{\Omega}_{1}, \boldsymbol{\Omega}_{2}\right)=$ $\frac{1}{\left|\Omega_{1}-\Omega_{2}\right|}$, namely

$$
V_{m_{1}^{\prime}, m_{2}^{\prime} ; m_{1}, m_{2}}=\int d \boldsymbol{\Omega}_{1} d \boldsymbol{\Omega}_{2} Y_{S, m_{1}^{\prime}}^{S}\left(\boldsymbol{\Omega}_{1}\right)^{*} Y_{S, m_{2}^{\prime}}^{S}\left(\boldsymbol{\Omega}_{2}\right)^{*} \frac{1}{\left|\boldsymbol{\Omega}_{1}-\boldsymbol{\Omega}_{2}\right|} Y_{S, m_{2}}^{S}\left(\boldsymbol{\Omega}_{2}\right) Y_{S, m_{1}}^{S}\left(\boldsymbol{\Omega}_{1}\right),
$$

where the vectors $\Omega$ are restricted to the surface of the sphere. The relevant matrix elements are calculated more explicitly in Ref. [5].

Now let us say a few words about the density operator in the spherical geometry. By definition,

$$
\rho(\boldsymbol{\Omega})=\sum_{i} \delta\left(\boldsymbol{\Omega}-\boldsymbol{\Omega}_{i}\right)
$$

where $\boldsymbol{\Omega}_{i}$ is the position of the $i^{\text {th }}$ electron. Since $\rho(\boldsymbol{\Omega})$ creates a neutral excitation when it acts on a state, it is useful to expand it in normal spherical harmonics, i.e. we define its Fourier components by

$$
\rho_{l m}(\boldsymbol{\Omega})=\sum_{i} Y_{l, m}\left(\boldsymbol{\Omega}_{i}\right)
$$

where $Y_{l, m}$ are the usual spherical harmonic (which are related to the monopole spherical harmonics [8] by $\left.Y_{l m}=Y_{l m}^{0}\right)$. It is easy to verify that $\rho_{l m}$ has the expected properties:

$$
\left[L_{z}, \rho_{l m}\right]=m \rho_{l m}, \quad\left[L_{ \pm}, \rho_{l m}\right]=\sqrt{l(l+1)-m(m \pm 1)} \rho_{l m \pm 1},
$$

and if $L^{2}|\Phi\rangle=0$, then

$$
L^{2} \rho_{l m}|\Phi\rangle=l(l+1) \rho_{l m}|\Phi\rangle, \quad \text { and } L_{z} \rho_{l m}|\Phi\rangle=m \rho_{l m}|\Phi\rangle
$$


In second quantized notation, we can write the density operator as

$$
\rho(\boldsymbol{\Omega})=\psi^{\dagger}(\boldsymbol{\Omega}) \psi(\boldsymbol{\Omega})
$$

where $\psi^{\dagger}(\boldsymbol{\Omega})$ and $\psi(\boldsymbol{\Omega})$ are the operators that create and annihilate respectively an electron at the point $\Omega$. As usual, the eigenstate operators $\left(C_{l, m}^{\dagger}\right)$ can be easily related to the position space operators $\left(\psi^{\dagger}(\boldsymbol{\Omega})\right)$ via

$$
C_{l, m}^{\dagger}=\int d \boldsymbol{\Omega} Y_{l m}^{S}(\boldsymbol{\Omega}) \psi^{\dagger}(\boldsymbol{\Omega})
$$

which can easily be inverted using the orthogonality property of the monopole spherical harmonics [8] to yield

$$
\psi^{\dagger}(\boldsymbol{\Omega})=\sum_{l, m}\left[Y_{l, m}^{S}(\boldsymbol{\Omega})\right]^{*} C_{l, m}^{\dagger}
$$

Thus we can write the density operator as

$$
\rho(\boldsymbol{\Omega})=\sum_{l_{1}, m_{1}} \sum_{l_{2}, m_{2}}\left[Y_{l_{1}, m_{1}}^{S}(\boldsymbol{\Omega})\right]^{*} Y_{l_{2}, m_{2}}^{S}(\boldsymbol{\Omega}) C_{l_{1}, m_{1}}^{\dagger} C_{l_{2}, m_{2}} .
$$

We can then take the angular momentum components to yield

$$
\rho_{l, m}=\int d \boldsymbol{\Omega} Y_{l, m}(\boldsymbol{\Omega}) \rho(\boldsymbol{\Omega})=\sum_{l_{1}, m_{1}} \sum_{l_{2}, m_{2}} \rho\left(l, m, l_{1}, m_{1}, l_{2}, m_{2}\right) C_{l_{1}, m_{1}}^{\dagger} C_{l_{2}, m_{2}}
$$

where

$$
\rho\left(l, m, l_{1}, m_{1}, l_{2}, m_{2}\right)=\int d \boldsymbol{\Omega} Y_{l, m}(\boldsymbol{\Omega})\left[Y_{l_{1}, m_{1}}^{S}(\boldsymbol{\Omega})\right]^{*} Y_{l_{2}, m_{2}}^{S}(\boldsymbol{\Omega})
$$

which is evaluated explicitly in Appendix D of Ref [1]. It is then easy to project this operator to the lowest Landau level $\left(l_{1}=l_{2}=S\right)$ to get

$$
\bar{\rho}_{l m}=\sum_{m^{\prime}} \bar{\rho}\left(l, m, m^{\prime}\right) C_{S, m+m^{\prime}}^{\dagger} C_{S, m^{\prime}},
$$

where

$$
\bar{\rho}\left(l, m, m^{\prime}\right)=(-1)^{S+l+m+m^{\prime}}(2 S+1) \sqrt{\frac{2 l+1}{4 \pi}}\left(\begin{array}{ccc}
S & S & l \\
-\left(m+m^{\prime}\right) & m^{\prime} & m
\end{array}\right)\left(\begin{array}{ccc}
S & S & l \\
-S & S & 0
\end{array}\right) .
$$


An important property of $\bar{\rho}_{l m}$ at $l=1$ is that

$$
\bar{\rho}_{1,0} \propto \bar{L}_{z}, \quad \bar{\rho}_{1,1} \propto \bar{L}_{+}, \quad \text { and } \bar{\rho}_{1,-1} \propto \bar{L}_{-},
$$

where the bar over the angular momentum operators indicate that they have been projected to the first Landau level also. This can be shown by writing the angular momentum operators in terms of eigenstate creation and annihilation operators [8], projecting to the first Landau level and comparing to the definition of the projected density operator. For example, we can write

$$
\bar{L}_{z}=\sum_{m} m C_{S, m}^{\dagger} C_{S, m}
$$

which is easily shown to be proportional to $\bar{\rho}_{1,0}$ once we have evaluated the $3-j$ coefficient in Eq. [17]. Using these properties (Eqs. [18]) of $\bar{\rho}_{1, m}$ we see that for $\left|\Phi_{0}\right\rangle$ in the first Landau level, $L^{2}\left|\Phi_{0}\right\rangle=0$ implies $\bar{\rho}_{1, m}\left|\Phi_{0}\right\rangle=0$; ie the projected density operator cannot generate excited states at $l=1$ from a uniform state.

Now we have come to the central quantity of our calculation. By definition, the projected dynamical structure factor is given by

$$
\bar{S}(l, \omega)=\sum_{\alpha}\left|\left\langle\Phi_{\alpha}\left|\bar{\rho}_{l m}\right| \Phi_{0}\right\rangle\right|^{2} \delta\left(\omega+\epsilon_{0}-\epsilon_{\alpha}\right),
$$

which is independent of $m$ by rotational invariance. By comparison, the full dynamical structure factor on a sphere is defined by

$$
S(l, \omega)=\sum_{\alpha}\left|\left\langle\Phi_{\alpha}\left|\rho_{l m}\right| \Phi_{0}\right\rangle\right|^{2} \delta\left(\omega+\epsilon_{0}-\epsilon_{\alpha}\right) .
$$

In the limit where the band mass is taken to zero, while the frequency $\omega$ and the electronelectron interaction are held fixed, the only energy states with finite $\epsilon_{\alpha}-\epsilon_{0}$ are states in which all electrons are restricted to the lowest Landau level. Thus if the frequency $\omega$ is fixed on the scale of the electron-electron interaction, the quantities $S(l, \omega)$ and $\bar{S}(l, \omega)$ become identical, as mentioned earlier. However, at energies on the scale of the cyclotron frequency, transitions between Landau levels will always be important, and the projected calculation 
will be inaccurate. In particular the projected structure factor will never display a Kohn mode and will never satisfy the $f$-sum rule.

We should also mention that for the exact diagonalization it is possible to take advantage of the rotational symmetry of the spherical system to reduce the dimension of the relevant many-body Hilbert space. Specifically, we explicitly block-diagonalize the Hamiltonian by computing its matrix elements between many-body states with the same total angular momentum $L^{2}$ and $L_{z}$. To construct an eigenstate of the total angular momentum operator $L^{2}$ with eigenvalue $l(l+1)$, we may in principle start from a randomly generated many-body state with the required $L_{z}$ and use the operator $\prod_{l^{\prime} \neq l}\left[L^{2}-l^{\prime}\left(l^{\prime}+1\right)\right]$ to project out the unwanted components with $L^{2}$ other than $l(l+1)$. However, on a finite precision computer, a naive application of this method is highly unstable, i.e. the error introduced by the machine round-off grows exponentially. Fortunately, there are ways to stabilize the algorithm so that eigenstates of $L^{2}$ with up to machine precision can be obtained. Details will be published elsewhere [10].

\section{B. Modified RPA}

As mentioned in the preceding companion paper [1], there are in principle two free parameters in the modified RPA. They are the ratio of the effective mass to the band mass $m^{*} / m_{b}$, and a constant $C=\left(\frac{\hbar^{2}}{m^{*} l_{0}^{2}}\right) /\left(\frac{e^{2}}{\epsilon l_{0}}\right)$, which is the ratio of the effective quasiparticle cyclotron energy to the Coulomb energy (which should be of order one). The estimate $C \approx 0.3$ has been made by Halperin, Lee, and Read [2] by examining results from exact diagonalizations of small systems. We will use this value of $C$ for all of our calculations in this paper.

In order to project our results to the lowest Landau level, we should take the band mass to zero, or $m^{*} / m_{b} \rightarrow \infty$. In practice, this is calculationally difficult, and we have actually used $m^{*} / m=50$ which is quite sufficient to separate the Kohn mode from the low energy

excitations. We then use the prescription described the preceding companion paper [1] to 
calculate the density-density response function $K_{00}(L, \omega)$ which is related to the dynamical structure factor via

$$
S(L, \omega)=\frac{-1}{\pi} \operatorname{Im}\left[K_{00}(L, \omega)\right]
$$

As noted in [可], the modified RPA is constructed to satisfy the $f$-sum rule, which one derives from the exact structure factor on the sphere. Within the units employed in this paper, this becomes

$$
\int_{0}^{\infty} d \omega \omega S(L, \omega)=\frac{L(L+1)}{8 \pi} \frac{N m^{*}}{m_{b}} \frac{C}{S}
$$

where the factor $\frac{C}{S}$ (with $S=\frac{N_{\phi}}{2}$ here) is simply a conversion of energy scales as described above. Note that this quantity diverges in the limit $m_{b} / m^{*} \rightarrow 0$. The main contribution to this sum rule, however, is from the Kohn mode at (or above) the cyclotron frequency. All other modes described by the modified RPA are low energy excitations (on the energy scale set by the Coulomb interaction). Moreover, as discussed above, in the exact diagonalizations, the projection to the lowest Landau level restricts our results to only those modes on the energy scale of the Coulomb interaction. Thus, in order to "project" $S(L, \omega)$ calculated in the modified RPA to the first Landau level, we simply discard the high frequency Kohn mode and call the result the projected dynamical structure factor $\bar{S}(L, \omega)$. We then define a projected contribution to the $f$-sum rule by

$$
\bar{f}(L)=\int_{0}^{\infty} d \omega \omega \bar{S}(L, \omega)
$$

The value of $\bar{f}(L)$ remains finite for $m_{b} / m^{*} \rightarrow 0$ for both the exact calculation and the modified RPA, so it is reasonable to try to compare the two.

In a similar manner, we define the projected static structure factor $\bar{S}(L)$, and the contribution $\Lambda(L)$ to the compressibility sum rule by

$$
\begin{gathered}
\bar{S}(L)=\int_{0}^{\infty} d \omega \bar{S}(L, \omega), \\
\Lambda(L)=\int_{0}^{\infty} d \omega \frac{\bar{S}(L, \omega)}{\omega} .
\end{gathered}
$$


In addition we shall report results for the average frequency entering in the single mode approximation (SMA) [9], which is simply defined by

$$
\omega_{L}=\frac{\int_{0}^{\infty} d \omega \omega \bar{S}(L, \omega)}{\int_{0}^{\infty} d \omega \bar{S}(L, \omega)}=\frac{\bar{f}(L)}{\bar{S}(L)} .
$$

The complete static structure factor $S(L)$ is defined using $S(L, \omega)$ on the right hand side of Eq. (25) instead of $\bar{S}(L, \omega)$. The contribution to $S(L)$ from the Kohn mode, or from inter-Landau-level transitions does not diverge in the limit $m_{b} / m^{*} \rightarrow 0$, but it is a large finite contribution; therefore, to make sensible comparisons between the modified RPA and the exact calculations for the lowest Landau level, we use the projected structure factor in both cases.

By contrast, in the limit $m_{b} / m^{*} \rightarrow 0$, the contribution to $\Lambda(L)$ from the Kohn mode or from inter-Landau-level transitions is vanishingly small. Therefore, it would not matter whether we use $\bar{S}(L, \omega)$ or $S(L, \omega)$ on the right hand side of Eq. (26).

\section{NUMERICAL RESULTS}

In this section we present and compare numerical results obtained from exact diagonalizations and from the modified RPA, for two filling factors in the principal sequence $\nu_{p}=\frac{p}{2 p+1}$. The first set of data, shown in Figs. 1 15, is obtained for a system of eight electrons at a filling factor $\nu=1 / 3$ (As required by Eqs. [1] and [2], $N_{\phi}=21$ here). In the composite fermion picture, at the mean field level, the groundstate of the system corresponds to a completely filled lowest effective Landau level for the composite fermions. The second set of data, shown in Figs. 6-10, is obtained for a system of twelve electrons at a filling factor $\nu=3 / 7\left(N_{\phi}=23\right)$, where the groundstate of the system corresponds to the lowest three filled effective Landau levels of the composite fermions.

As discussed in the Refs. [1]:2], and [3], in a planar system, we expect the modified RPA to be more accurate in the large $p$ limit (ie, $\nu$ approaching $1 / 2$ ) where the motion

of the composite fermions is semiclassical. In the present calculations however, we do not 
necessarily expect the modified RPA to be more accurate for the $\nu=3 / 7(p=3)$ state than for the $\nu=1 / 3(p=1)$ state due to the problem of enhanced finite size effects for the $\nu=3 / 7$ state. Although the $\nu=3 / 7$ system contains more electrons $(N=12)$ than the $\nu=1 / 3$ system $(N=8)$, it should be considered the "smaller" system in that the composite fermion magnetic length is much larger in the $\nu=3 / 7$ system, and the number of electrons per Landau level is also smaller. Thus, any finite size effects will be accentuated in the $\nu=3 / 7$ set of data, and we suspect that the modified RPA will be less accurate in this case.

In Fig. 1, we show the projected spectral weights of $\bar{S}(L, \omega)$ for a system of eight electrons at a filling factor $\nu=1 / 3\left(N_{\phi}=21\right)$, calculated through exact diagonalization (upper graph) and using the modified RPA (lower graph). The weight of each delta function contribution to $\bar{S}(L, \omega)$ at each $(L, \omega)$ is proportional to the area of the corresponding shaded rectangle. Here and in the rest of this work, the energy scale is given in units of $e^{2} /\left(\epsilon l_{0}^{2}\right)$. As we discussed above, although there may be excitations at $L=1$, these states all have zero weight in the projected dynamical structure factor.

We see immediately from Fig. 1 that the projected dynamical structure factor calculated in the modified RPA agrees very well with the exact diagonalization at low energies. In particular, the distribution of spectral weights among the states in the lowest collective mode is very similar in the upper and lower graph. The small discrepancy in overall energy scales might be due to an inaccuracy in our guess of the energy scale conversion factor $C$ described above.

We note that the lowest collective mode dominates the spectral weight for angular momenta up to $L=L_{\text {roton }} \approx 4$ in the exact diagonalization, particularly near the roton minimum $L_{\text {roton. }}$ In the modified RPA the lowest mode dominates only at smaller angular momenta. At higher angular momenta $\left(L>L_{\text {roton }}\right)$, the exact diagonalization shows a fairly significant amount of spectral weight at energies $E \sim 2 E_{\text {roton }}$. It is believed that the corresponding states can roughly be represented as composite objects of two rotons [11]. We should not expect that such states would be properly modeled in the modified RPA since the RPA only represents single quasiparticle-quasihole excitations. However, as long as we 
consider sufficiently low energy (not too much larger than $E \sim 2 E_{\text {roton }}$ ), the modified RPA seems to agree very well with the exact diagonalization.

At higher energies, in the continuum of the exact diagonalization spectra, we empirically observe that the spectral weights decay exponentially $\bar{S}(L, \omega) \sim e^{-\omega / \Gamma}$ for a given $L$, where $\Gamma \sim 0.03 e^{2} / \epsilon l_{0}$. In this high energy region, where the modified RPA differs significantly from the exact results, we see that the modified RPA yields a discreet spectrum of high energy modes with a large amount of spectral weight. The discreteness of the modified RPA is an obvious artifact arising from the neglect of processes where a higher energy mode can decay into several low energy modes with the same total energy and wavevector. The fact that the modified RPA seriously overestimates the total weight at high energies is a more significant limitation of the approximation.

In Fig. 2 we show unweighted excitation spectra for the same system of $N=8$ electrons at $\nu=1 / 3$. The upper graph is the complete energy spectrum in the range $0 \leq \omega<0.45$, $0 \leq L<11$, from exact diagonalization regardless of whether or not the state contributes to $\bar{S}(q, \omega)$. The lower graph is again the result of the modified RPA. Note that the dispersion relations and the overall energy scale of the lowest collective mode are very similar in the two cases. The black circles in the two graphs are the spectra of the single mode approximation (SMA) computed from with Eq. [27], for the exact calculations and modified RPA respectively. We observe that in the exact diagonalization results, the SMA works well for angular momenta up to the roton minimum $L_{\text {roton }}$ while in the modified RPA, the SMA fails at all but the lowest angular momentum. This failure of the SMA is due to the erroneous predictions of the modified RPA at high energy. In the modified RPA, as we increase the angular momentum, the main contribution to the spectral weight comes from a frequency that increases approximately proportionally to $L(L+1)$. This can be explained roughly by considering the modified RPA as a perturbation of a free electron system where it is well known that the excitations are confined to a band whose upper and lower energy boundaries both vary as $q^{2}$. When a magnetic field is added to such a free electron system, a Kohn mode appears, and some of the low energy excitations can be changed, but we expect that most 
of the high $q$ excitations remains roughly in the same band. As discussed above, the single particle nature of the modified RPA allows these high energy modes to be given too much spectral weight. Thus we see how the the single mode approximation in the modified RPA is dominated by the poorly modeled higher frequencies in the modified RPA, thus giving very inaccurate results.

In Fig. 3, we show the projected static structure factor $\bar{S}(L)$ (see Eq. [25]) for the same system of $N=8$ electrons at $\nu=1 / 3$. The circles (solid line) are from the exact diagonalization whereas the triangles (dotted line) are the result of the modified RPA. The inset is the same modified RPA data on a reduced scale to more clearly show the behavior at higher angular momenta. Of course the projected static structure factor is only defined at integer values of $L$, and the lines (dotted and solid) are just guides for the eye. In the exact diagonalization (circles), we find that $\bar{S}(L)$ peaks around the roton minimum and decreases rapidly at higher angular momenta. We have studied its behavior and found empirically that it roughly obeys a Gaussian $\bar{S}(L) \sim e^{-\alpha L(L+1)}$, where $\alpha$ is a constant. This reflects a similar empirically observed Gaussian decay of the matrix elements of the density operator at large angular momentum. The projected static structure factor in the modified RPA (triangles) is very similar to the exact result (circles) at small values of the angular momentum where the modified RPA does not predict extraneous high energy modes with large amounts of weight. At higher values of $L$ the erroneous high energy modes cause a severe overestimation of the projected static structure factor.

In Figs. 1 and 5, we show the contribution to the $f$-sum rule and compressibility sum rule as defined in Eqs. [24 and 26] for the same system of $N=8$ electrons at filling fraction $\nu=1 / 3$. As above, the circles are from the exact diagonalization whereas the triangles are the result of the modified RPA, and the lines are guides for the eye. Once again the insets show the same modified RPA data on a reduced scale. In Fig. 田, we see that the contribution to the $f$-sum rule suffers from the same erroneous high energy modes. The compressibility sum rule (Fig. 5) is somewhat better estimated by the modified RPA because the contribution of the erroneous high energy modes are suppressed by a factor of 
$\omega$ in this sum rule (see Eq. [26]). This is somewhat encouraging, since the compressibility sum is the most relevant of these quantities for describing the low energy behavior of the quantized Hall state.

In Fig. 6, we show the projected dynamical structure factor $\bar{S}(L, \omega)$ for a system of $N=12$ electrons at a filling factor $\nu=3 / 7\left(N_{\phi}=23\right)$. Again, the spectral weights of $\bar{S}(L, \omega)$ at each $(L, \omega)$ are proportional to the area of the corresponding shaded rectangles. We observe again that the behaviors of $\bar{S}(L, \omega)$ from exact diagonalization (upper graph) and calculated in the modified RPA (lower graph) are semi-quantitatively similar. However, once again, the modified RPA results clearly overestimate the spectral weights at high energies.

In Fig. 7, we show the corresponding complete excitation spectra of the system of $N=12$ electrons at a filling factor $\nu=3 / 7$. An interesting feature to note is that there are wiggles in the dispersion of the lowest collective mode in both the exact calculation and the modified RPA. Note particularly how the lowest energies at $L=3$ and 5 lie above the lowest energies at $L=2,4$, and 6 . This behavior was observed previously in the exact calculation for this system size and filling factor by d'Ambrumenil and Morf [6]. These authors posed the question whether the even-odd alternation might be a spurious effect due to the finite size of the system. Based on the modified RPA analysis, however, we believe that the maxima and minima in the spectrum are genuine effects reflecting the maxima and minima that were previously found [3] in the dispersion relation for the lowest excitation branch in the planar system using this approximation. More generally, for filling fractions of the form $\nu=p /(2 p+1)$, at large values of $p$, it was predicted in Ref. [3] that there should be a series of maxima and minima in the dispersion of the lowest excitation branch occuring respectively at wavevectors of the form $q=\pi\left(n-\frac{1}{4}\right) / R_{c}^{*}$ and $q=\pi\left(n+\frac{1}{4}\right) / R_{c}^{*}$, where $n$ is a positive integer, and $R_{c}^{*}$ is the "effective cyclotron radius" for the composite fermions. This quantity is given by the relation

$$
R_{c}^{*}=\hbar k_{\mathrm{F}} /(e \Delta B)=2 p / k_{\mathrm{F}},
$$

where $\Delta B$ is the deviation of the magnetic field from the field at $\nu=1 / 2$, and the Fermi 
wavevector $k_{\mathrm{F}}$ is related to the electron density $n_{e}$ by $k_{\mathrm{F}}=\left(4 \pi n_{e}\right)^{1 / 2}$. Thus, at filling fraction $\nu=p /(2 p+1)$, we have

$$
R_{c}^{*}=\frac{1}{l_{0}}(2 p)^{1 / 2}(2 p+1)^{1 / 2}
$$

If we now apply this formula to our system of particles on a sphere, with $l_{0}^{-1}=\left(N_{\phi} / 2\right)^{\frac{1}{2}}$, we find $R_{c}^{*} \approx 2$ at $\nu=3 / 7$, with $N=12$. Thus one might expect maxima and minima to alternate with a period of $\Delta L=\pi / R_{c}^{*} \approx 1.6$. Since the observed alternation with a period of $\Delta L=2$ is not far from this expectation, we believe it is appropriate to identify the oscillations as the expression in our finite size system of the oscillations predicted for the plane. In any case, the fact that this detail of the spectrum predicted from the modified RPA for the finite system is in good agreement with the exact calculations gives support to the belief that the oscillations predicted for the infinite system should also be present in an exact calculation.

The black circles in Fig. [0 show the single mode approximation (Eq. [27]) for $\nu=3 / 7$. As we discussed in the case of $\nu=1 / 3$, the single mode approximation clearly shows that the modified RPA predicts too much spectral weight at high energies.

In Figs. 8, 9, and 10, we show the projected static structure factor (Eq. [25]), the contribution to the $f$-sum rule (Eq. 24]) and the contribution to the compressibility sum rule (Eq. [26]) for the same system of $N=12$ electrons at filling fraction $\nu=3 / 7$. As above, the circles are always from the exact diagonalization and the triangles are from the modified RPA. Once again the insets show the modified RPA data on a reduced scale to show the behavior at higher angular momenta. Again, we see that the erroneous high energy modes in the modified RPA dominate the $f$-sum and the static structure factor at all but the lowest angular momenta. On the other hand, the compressibility sum (Fig. 10) is somewhat better represented in the modified RPA due to the suppression of these high energy modes. 


\section{CONCLUSIONS}

We have studied the excitation spectra and the dynamical structure factor in quantum Hall states belonging to the principal sequence $\nu=p /(2 p+1)$ through exact diagonalization and projected modified RPA. In particular, we have presented results from finite size exact diagonalization and from projected modified RPA on a system of $N=8$ electrons at $\nu=1 / 3$ and a system of $N=12$ electrons at $\nu=3 / 7$. We find that the modified RPA works

reasonably well at low energies, ie for energies $\lesssim 0.2$, in units of $e^{2} / \epsilon l_{0}$. (By comparison, the effective Fermi energy $E_{\mathrm{F}}=k_{\mathrm{F}}^{2} /\left(2 m^{*}\right)$ is approximately $C / 2 \approx 0.15$, in these units.)

By combining our results with previous analyses, we can arrive at some reasonable conjectures for the excitation spectrum in a planar system, which should hold in particular for relatively large values of the parameter $p$.

1. The present work supports the conjecture that the modified RPA gives a qualitatively correct description of the dispersion curve for the lowest branch of the excitation spectrum (quasiexciton mode) at $\nu=p /(2 p+1)$. The series of maxima and minima predicted by the modified RPA in the planar limit have analogs in the finite system which are also observed in the exact calculations.

2. The general distribution of the spectral weight $S(q, \omega)$ for frequencies above the lowest excitation branch, up to energies of the order of $\omega \approx 0.2 e^{2} /\left(\epsilon l_{0}\right)$, for wavevectors in the range $0 \leq q \leq 2 k_{\mathrm{F}}$ is likely to be represented in a qualitatively correct fashion by the modified RPA. Of course certain details must clearly be wrong as has been previously discussed [3]. Higher branches of the energy spectrum, which are undamped in the modified RPA, should actually be broadened into a continuum by decay into multiple excitations of lower lying modes.

3. The modified RPA predicts that for large values of $p$, and wavevectors in the range $k_{\mathrm{F}} / p \ll q \ll k_{\mathrm{F}}$, the dominant contributions to the static compressibility sum rule should arise from frequencies $\omega \propto q^{2}$. [2] We believe this prediction to be correct, but our finite size systems are too small to give any direct confirmation. 
4. The modified RPA predicts that there should be sizable contributions to $\bar{S}(q, \omega)$, for $\omega \gtrsim 0.2 e^{2} /\left(\epsilon l_{0}\right)$, for large wavevectors in the range where $\left|q^{2}-2 m^{*} \omega\right| \lesssim 2 k_{\mathrm{F}} q$. We believe these predictions to be spurious, however, as exact finite size system calculations show little weight in $\bar{S}(q, \omega)$ at high frequencies for any value of $q$. Of course the complete dynamical structure factor $S(q, \omega)$ will contain additional contributions at very high frequencies arising from transitions between Landau levels.

5. The above conjectures are of course predicated on the assumption that no instability or phase transition occurs at very large values of $p$, which would then invalidate our analysis. Finite size system calculations cannot rule out the possibility of such an instability at large $p$.

\section{ACKNOWLEDGMENTS}

The work at Harvard was supported by the National Science Foundation Grant DMR91-15491. 


\section{REFERENCES}

[1] S. H. Simon and B. I. Halperin, preceding paper Response Function of the Fractional Quantized Hall State on a Sphere I: Fermion Chern-Simons Theory. In this reference the density-density response function $K_{00}(L, \omega)$ is calculated, which is related to the dynamical structure factor via Eq. [22].

[2] B. I. Halperin, P. A. Lee, and N. Read, Phys. Rev. B47, 7312(1993), and references therein.

[3] S. H. Simon and B. I. Halperin, Phys. Rev. B 48, 17386 (1993).

[4] F. D. M. Haldane, p.303, in "The Quantum Hall Effect," Eds. R. E. Prange and S. M. Girvin (Springer, New York, 1987).

[5] G. Fano, F. Ortolani, and E. Colombo, Phys. Rev. B34, 2670(1986).

[6] N. d'Ambrumenil and R. Morf, Phys. Rev. B40, 6108 (1989); see also A. M. Reynolds and N. d'Ambrumenil, J. Phys. C21 5643 (1988).

[7] X. G. Wen and A. Zee, Phys. Rev. Lett. 69, 953 (1992).

[8] T. T. Wu and C. N. Yang, Nucl. Phys. B107, 365 (1976).

[9] S. M. Girvin, A. H. MacDonald, and P. M. Platzman, Phys. Rev. B33 2481 (1986).

[10] Song He, unpublished.

[11] Song He and P. M. Platzman, submitted to Phys. Rev. B. 


\section{FIGURES}

FIG. 1.

The weights of the projected dynamical structure factor $\bar{S}(L, \omega)$ for $N=8$ electrons at filling fraction $\nu=1 / 3\left(N_{\phi}=21\right)$. The upper graph is obtained from exact diagonalization, and the lower graph is obtained from modified RPA. The magnitude of each delta function contribution to the projected dynamical structure factor $\bar{S}(L, \omega)$ is proportional to the area of the corresponding shaded rectangle.

FIG. 2.

The excitation spectra for $N=8$ electrons at filling fraction $\nu=1 / 3$. The upper graph is obtained from finite size exact diagonalization, and the lower graph is obtained from modified RPA. The black circles are spectra calculated in the single mode approximation (see Eq. [27]).

FIG. 3.

The projected static structure factor $\bar{S}(L)$ (See Eq. 25]) for $N=8$ electrons at filling fraction $\nu=1 / 3$. The circles are obtained from finite size exact diagonalization, and the triangles are obtained from the modified RPA. The inset is the modified RPA data on an expanded scale to show the behavior at large $L$. The solid and dotted lines are guides for the eye.

FIG. 4. 
The contribution to the $f$-sum rule $\bar{f}(L)$ (See Eq. [24]) for $N=8$ electrons at filling fraction $\nu=1 / 3$. The circles are obtained from finite size exact diagonalization, and the triangles are obtained from the modified RPA. The inset is the modified RPA data on an expanded scale to show the behavior at large $L$. The solid and dotted lines are guides for the eye.

FIG. 5 .

The contribution to the compressibility sum rule $\Lambda(L)$ (See Eq. [26]) for $N=8$ electrons at filling fraction $\nu=1 / 3$. The circles are obtained from finite size exact diagonalization, and the triangles are obtained from the modified RPA. The inset is the modified RPA data on an expanded scale to show the behavior at large $L$. The solid and dotted lines are guides for the eye.

FIG. 6.

The projected dynamical structure factor $\bar{S}(L, \omega)$ for $N=12$ electrons at filling fraction $\nu=3 / 7\left(N_{\phi}=23\right)$. The upper graph is obtained from exact diagonalization, and the lower graph is obtained from modified RPA. The magnitude of each delta function contribution to the projected dynamical structure factor $\bar{S}(L, \omega)$ is proportional to the area of the corresponding shaded rectangle.

FIG. 7. 
The excitation spectra for $N=12$ electrons at filling fraction $\nu=3 / 7$. The upper graph is obtained from finite size exact diagonalization, and the lower graph is obtained from modified RPA. The black circles are spectra calculated in the single mode approximation (see Eq. [27])

FIG. 8.

The projected static structure factor $\bar{S}(L)$ (See Eq. [25]) for $N=12$ electrons at filling fraction $\nu=3 / 7$. The circles are obtained from finite size exact diagonalization, and the triangles are obtained from the modified RPA. The inset is the modified RPA data on an expanded scale to show the behavior at large $L$. The solid and dotted lines are guides for the eye.

FIG. 9 .

The contribution to the $f$-sum rule $\bar{f}(L)$ (See Eq. [24]) for $N=12$ electrons at filling fraction $\nu=3 / 7$. The circles are obtained from finite size exact diagonalization, and the triangles are obtained from the modified RPA. The inset is the modified RPA data on an expanded scale to show the behavior at large $L$. The solid and dotted lines are guides for the eye.

FIG. 10. 
The contribution to the compressibility sum rule $\Lambda(L)$ (See Eq. [26]) for $N=12$ electrons at filling fraction $\nu=3 / 7$. The circles are obtained from finite size exact diagonalization, and the triangles are obtained from the modified RPA. The inset is the modified RPA data on an expanded scale to show the behavior at large $L$. The solid and dotted lines are guides for the eye. 